4. Gong AK, Van Heuven WAJ, Berlanga M, Escobedo MB. Severe retinopathy in convalescent preterm infants with mild or regressing retinopathy of prematurity. Paediatrics 1989;83:422-5.

5. Ferrone PJ, de Juan E. Vitreous haemorrhage in infants. Arch Ophthalmol 1994;112:1185-9.

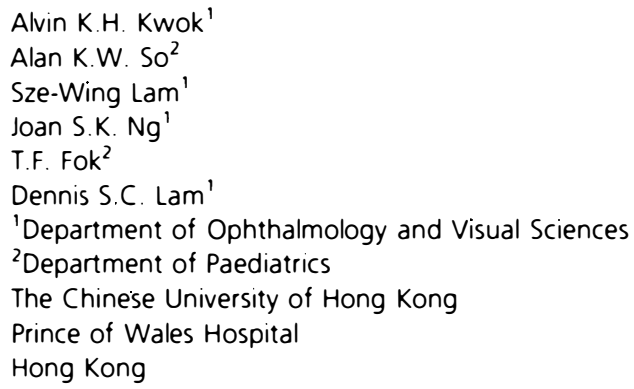

Sir,

\section{Nightmares with topical beta-blocker}

The beta-blockers have been the first line of treatment for glaucoma for many years and the standard against which all other treatments have been compared for their pressure-lowering effects. Timolol, like all beta-blockers, has well-known cardiovascular and respiratory side effects and should be prescribed very carefully, especially in elderly patients with diseases affecting these systems. A small minority of patient get central nervous system (CNS) side effects with topical beta-blockers, which have mainly been reported with oral betablockers. ${ }^{1}$ These include behavioural changes such as confusion, hallucinations, anxiety, disorientation, nervousness, somnolence and other psychiatric disturbances. Nightmares have been reported commonly with oral beta-blockade but, as far as we know, there has been only one previous case published of nightmares with topical ophthalmic beta-blockers and unlike that case our patient experienced these despite the regular practice of punctal occlusion.

\section{Case report}

A 61-year-old man diagnosed to have open angle glaucoma with intraocular pressures of 28 and $26 \mathrm{mmHg}$ in the right and left eye respectively was started on brimonidine $0.2 \%$ drops but did not tolerate them very well and had an allergic reaction together with stinging on instillation. His past medical history was unremarkable and he was not on any systemic medications at the time of commencement of the topical treatment. As there were no obvious contraindications to the use of beta-blockers he was commenced on $0.5 \%$ timolol maleate drops but returned within 3 weeks as he was feeling very tired and lethargic and was having disturbing nightmares. The drops were being instilled at 0800 hours in the morning and at 2000 hours at night with the patient occluding the lacrimal punctum for $30 \mathrm{~s}$ afterwards. The nightmares had begun within a week of the treatment and recurred every night, they were obtrusive in nature and the content was more or less the same with detailed recall of the events. The physical examination and a Mini Mental State Examination were normal, and the results of his recent routine laboratory tests including blood glucose, full blood counts, blood urea and electrolytes were within normal limits. There were no other behavioural or psychiatric disturbances and, at this point, as his nightmares could not be linked to his topical medication it was decided to persist with timolol, but the patient came back within a week and was now very distressed by the nightmares. At this point it was decided to change the eyedrops to pilocarpine $2 \%$ three times a day. The nightmares stopped promptly and have not returned over the last 6 months while the intraocular pressure is well controlled at $18 \mathrm{mmHg}$ in both eyes.

\section{Comment}

A wide variety of adverse central nervous system (CNS) effects have been reported with beta-blockers including bizarre dreams, nightmares, depression, dizziness, tinnitus, headache, somnolence, inability to concentrate and insomnia. ${ }^{1-3}$ They occur either immediately after starting therapy or may be delayed for many years and are mainly seen with oral administration. These side effects are mainly dependent on the plasma concentrations, lipid solubility, protein binding and the volume of distribution of the drug. Topical medications can reach significant concentrations in the systemic circulation mainly by absorption through conjunctival veins, lacrimal passages and the vascular nasal mucosa, and the serum concentrations of timolol have been shown to reach levels of $1.39 \mathrm{ng} / \mathrm{ml} 1 \mathrm{~h}$ after ophthalmic administration, ${ }^{4}$ sufficient to cause systemic adverse effects. The various methods described to reduce systemic levels, including closure of the eyelids and lacrimal occlusion, have been shown to reduce the serum levels by up to $65 \%{ }^{5}$ but may still be inadequate to prevent systemic side effects including CNS effects. The brain primarily contains $\beta 1$ receptors and, depending on their lipid solubility, the beta-blockers may variably block them, but the exact mechanism for the potential CNS and psychiatric side effects with beta-blockers is still not clear and has been ascribed to depressed melatonin levels in these patients. ${ }^{6}$ Only a very small number of individual case reports of ophthalmic medications causing CNS adverse effects have appeared in the literature and only one of nightmares with betaxolol, ${ }^{7}$ but unlike our patient, the nightmares were stopped by punctal occlusion. In our patient, the onset of nightmares with timolol, absence of other possible 
contributory factors and the prompt cessation of the nightmares on stopping timolol indicates the drops as the most probable cause.

Busy outpatient clinics may not allow sufficient time to enquire about these less well known adverse effects, which may result in unnecessary investigations in some elderly patients to rule out psychiatric disorders. An awareness of the possible CNS side effects on the part of general practitioners and treating ophthalmologists may save precious time and resources for the health system and avoid anxiety for the patient.

\section{References}

1. Westerlund A. Central nervous system side effects with hydrophilic and lipophilic beta-blockers. Eur J Clin Pharmacol 1985;28(Suppl):73-6.

2. Munroe WP, Rindone JP, Kreshner RM. Systemic side effects associated with the ophthalmic administration of timolol. Drug Intell Clin Pharm 1985;19:85-9.

3. Zimmerman TJ, Baumann JD, Hetherington J. Side effects of timolol. Surv Ophthalmol 1983;28(Suppl):243-9.

4. Passo MS, Palmer EA, Van Buskirk EM. Plasma timolol in glaucoma patients. Ophthalmology 1984;91:1361-3.

5. Zimmerman TJ, Kooner KS, Kandarkis AS, Zeigler LP. Improving the therapeutic index of topically applied ocular drugs. Arch Ophthalmol 1984;102:551-3.

6. Brismer K, Mogensen L, Wetterberg L. Depressed melatonin secretion in patients with nightmares due to betaadrenoceptor blocking drugs. Acta Med Scand 1987;221:155-8

7. Mort JR. Nightmare cessation following alteration of ophthalmic administration of a cholinergic and a betablocking agent. Ann Pharmacother 1992;626:914-6.

A. Negi

D. Thoung

F. Dabbous

Broomfield Hospital

Chelmsford, UK

Mr A. Negı, MD, FRCSEd, MRCOphth

Department of Ophthalmology

Leeds General Infirmary

St. George Street

Leeds LS1 3EX, UK

\section{Sir}

The importance of recognising Streptococcus milleri as a cause of orbital cellulitis

We present three cases of orbital cellulitis caused by Streptococcus milleri (S. milleri) which highlight the organism's propensity for abscess formation, necessitating surgical drainage and a long course of antibiotic therapy.

\section{Case reports}

Case 1. A 15-year-old boy presented with marked inflammation of the preseptal tissues and restriction of the extraocular movements. A CT scan showed an opaque ethmoid and maxillary sinus with a subperiosteal abscess. Intravenous co-amoxiclav (Augmentin, Beecham) $1.2 \mathrm{~g}$ t.d.s. was commenced and ethmoidectomy and drainage of subperiosteal abscess was performed. Following an initial improvement, the periorbital swelling increased. Further surgical exploration and drainage of pus was performed. The patient improved once again and was discharged home 4 days later on oral Augmentin $625 \mathrm{mg}$ PO t.d.s for 10 days. Four days following the completion of the oral antibiotics the patient was readmitted with a recurrence of the orbital cellulitis. An MRI scan showed a large soft tissue mass in the roof of the orbit (Fig. 1). A frontal sinus pus collection and a subperiosteal abscess were drained and intravenous antibiotics restarted. Streptococcus constellatus was cultured from pus swabs from both drainage procedures. The patient was discharged home on a 6 week course of oral Augmentin and his further recovery was uneventful.

Case 2. A 31-year-old man presented with a 1 day history of left periorbital swelling, proptosis and diplopia. A CT scan showed an opaque maxillary sinus. He was admitted and given intravenous cefotaxime and metronidazole. A left inferior meatal antrostomy and pus drainage was performed. Following an initial improvement the proptosis increased. An inferior subperiosteal abscess was drained. The signs improved and 4 days later the patient was discharged. S. milleri was cultured from a pus swab from the second drainage procedure. The patient received a 6 week course of oral Augmentin. There were no further complications or relapses.

Case 3. A 12-year-old boy presented with left periorbital swelling and pyrexia. He was admitted and commenced on intravenous Augmentin. The following day the patient underwent an antral washout and aspiration of pus. He failed to improve and 2 days later he underwent

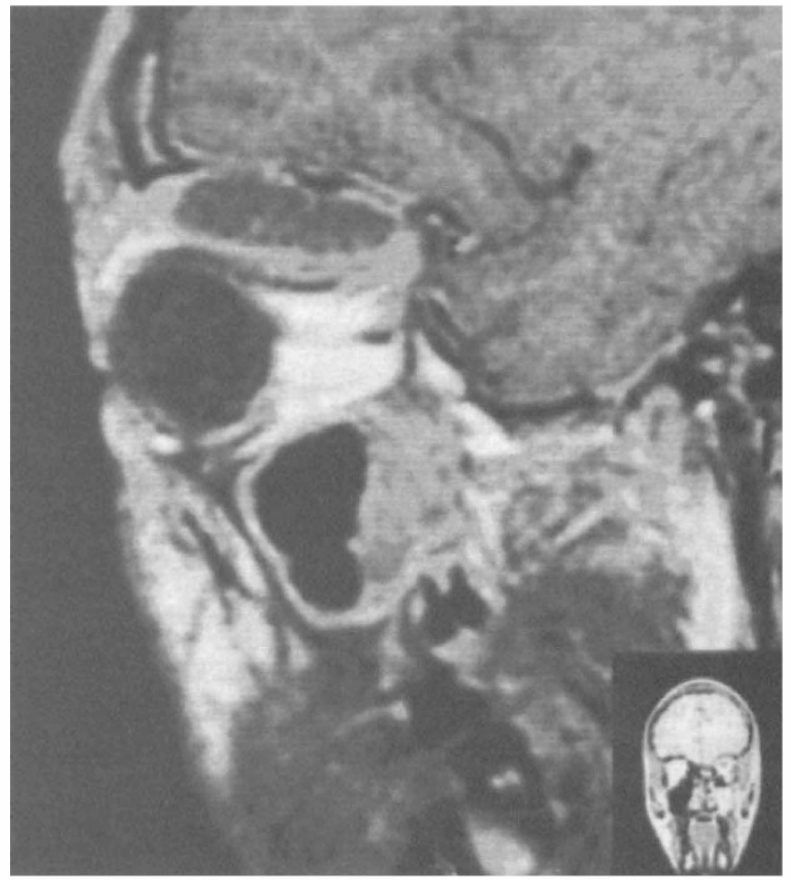

Fig. 1. Case 1. A sagittal MRI image through the left orbit showing a superior orbital collection. 